\title{
Explicating Universal Design for Learning-aligned Instructional Practices for Postsecondary STEM
}

\author{
Jacquelyn J. Chini \\ Department of Physics, University of Central Florida, 4111 Libra Dr., Orlando, FL, 32816 \\ Erin K. H. Saitta \\ Department of Chemistry, University of Central Florida, 4111 Libra Dr., Orlando, FL, 32816 \\ Abdelkader Kara \\ Department of Physics, University of Central Florida, 4111 Libra Dr., Orlando, FL, 32816 \\ Erin Scanlon \\ Department of Physics, University of Connecticut - Avery Point, Groton, CT 06340
}

\begin{abstract}
Postsecondary STEM instruction often does not meet the diverse needs, abilities, and interests of postsecondary STEM learners. The Universal Design for Learning (UDL) framework provides ideas for designing instructional environments to support learner variation. However, existing tools do not meet the professional development needs of many postsecondary STEM instructors, or the research needs of some discipline-based education researchers. Enacting and assessing UDL in postsecondary STEM requires expertise form three stakeholder groups: postsecondary STEM instructors, experts in disability and UDL, and disciplinebased education researchers. Building on prior work with UDL in postsecondary STEM, our multi-disciplinary team developed the Universal Design for Learning Instructional Practice Observation Protocol (UDL-IPOP). The UDL-IPOP has one-to-one alignment with the finest-grain descriptors (i.e., checkpoints) in the UDL framework as well as exemplar practices to explicate UDL-aligned instructional practices for postsecondary STEM. After drafting an initial version of the UDL-IPOP, we discussed it with representatives from the three relevant stakeholder groups. Here, we describe practices that aligned with the UDL framework. Then, we discuss initial impressions about which practices postsecondary STEM instructors likely already implement and ideas from disability experts about how STEM instructors could deepen their UDL practice. Overall, we find that postsecondary STEM instructors and disability experts focused on different types of learner variation. We suggest that collaborating with disability experts could provide a necessary "lens change" to better support variation in postsecondary STEM learners' needs, abilities, and interests.
\end{abstract}

2021 PERC Proceedings edited by Bennett, Frank, and Vieyra; Peer-reviewed, doi.org/10.1119/perc.2021.pr.Chini

Published by the American Association of Physics Teachers under a Creative Commons Attribution 4.0 license.

Further distribution must maintain the cover page and attribution to the article's authors. 


\section{INTRODUCTION}

All students vary in their needs, abilities, and interests. Universal Design for Learning (UDL) is a framework to support proactive instructional design (e.g., curricular materials, room layouts, instructional activities, course policies) that plans for the needs of all learners [1]. Postsecondary instructors often do not know how to provide support for some types of learner variation [2-8], and STEM instructors have demonstrated more negative views towards disabled students than instructors in other disciplines [9-12]. While postsecondary instructors typically do not know in advance the learner variation represented in a class, tools exist to support instructors in considering the dimensions of ability along which individuals vary [13-14].

Research with a survey of instructors' beliefs about and usage of inclusive teaching strategies suggests that postsecondary physics instructors have beliefs about teaching that are more inclusive than their actions [15]. Thus, we want to develop a tool to support postsecondary STEM instructors to shift from belief to action by implementing more accessible and inclusive practices. Ideally, this tool would also be useful for evaluating change in instructional practice. In this paper, we present the initial development process of the Universal Design for Learning Instructional Practice Observation Protocol (UDL-IPOP) to describe UDL-aligned instructional practices in the postsecondary STEM context and to discuss the findings of interviews with three stakeholder groups about the UDL-IPOP.

\section{PRIOR WORK OBSERVING UDL}

UDL is described by three principles: provide multiple means of 1) representation, 2) action and expression, and 3) engagement. Each principle is further described by three guidelines per principle and several checkpoints [1].

Our research team has worked with postsecondary physics and chemistry instructors to apply UDL to proactively design supports for variation in students' needs, abilities, and interests (Project ACCESSS). Our process involved observing class sessions, providing feedback, engaging instructors in professional development about UDL and disability, and supporting instructors to develop and implement UDL-aligned strategies [16-17].

UDL is challenging to measure as it is a framework for design rather than a specific practice [18]. We used an earlier version of the UDL Observation Measurement Tool (UDLOMT, a protocol validated in K-12 settings) in Project ACCESSS, however we found that this and other extant tools for observations and professional development did not meet the needs of postsecondary STEM instructors or our needs as discipline-based education researchers. For example, the UDL-OMT does not have a one-to-one alignment with the UDL framework, is structured based on when the developers thought checkpoints would likely be observed during a typical K-12 lesson, focuses only on what happens during class, and is based on a three-point scale that was difficult to operationalize in the context of postsecondary STEM. Thus, our team began to document the practices they observed during a class session that aligned with a particular item, expanding the protocol to include example practices. Since few other tools exist for guiding observations of UDL (i.e., [19]), we decided to design a tool that met our goal of demonstrating evidence-based practices to support learner variation in postsecondary STEM and used these practices as a starting point.

\section{UDL-IPOP DEVELOPMENT PROCESS}

Our goal of demonstrating evidence-based practices for supporting learner variation in postsecondary STEM requires the expertise of researchers in postsecondary STEM discipline-based education research (DBER), researchers in disability and special/exceptional education with UDL expertise, postsecondary STEM instructors, and disabled STEM students. Our research and development team (representing chemistry, physics, and exceptional education) includes participants from the first three of these communities; we acknowledge the need to collaborate with and center students' voices (e.g., Refs. 20-21). We cocreated goals for the protocol, including that the protocol should: 1) be useful for researchers and STEM instructors; 2) describe the UDL-aligned strategies that instructors implement in a class session; and 3) demonstrate variation in implementation of UDL-aligned strategies over a class period, across instructors, and over time. We envisioned that the protocol would focus on in-class components with a complementary survey to measure out-of-class components. Based on these goals, we expected that an interval-based protocol with a one-to-one correlation with UDL checkpoints would best meet our needs.

In line with our goals, each UDL checkpoint was phrased as an observation item. The UDL checkpoints were housed within the broader UDL principles and guidelines. We used the results of our prior research on UDL in postsecondary STEM (e.g., Refs. 20-23) to add exemplar practices for each checkpoint as a starting point for discussion. Then, the interdisciplinary team met and discussed each checkpoint, which resulted in changes to the wording of the practices to be understandable and meaningful to all three groups, and the addition of practices based on individuals' experiences.

\section{METHODS}

We recruited participants from three stakeholder populations: postsecondary STEM instructors, DBER, and researchers in exceptional education experienced with UDL. We interviewed ten STEM instructors (representing chemistry (3), physics (4), biology (1) and mathematics (2)), four of whom also identified with DBER, and four researchers in exceptional education and UDL; in Section V participants are referred to by their discipline and a number 
TABLE I. Strategies to Provide Multiple Means of Representation

\begin{tabular}{l}
\hline Guideline 1: Options for perception \\
\hline 1A Information was available digitally \\
1B Visual presentation allowed for customization \\
1C Used visual aids as alternative to auditory information \\
1D Closed captions for videos and discussion \\
1E Material was described verbally \\
1F Alternative text was provided for visual information \\
\hline Guideline 2: Options for language, mathematical \\
$\quad$ expression, and symbols \\
\hline 2A Symbols were named and described \\
2B Provided descriptions of vocabulary \\
2C Explained how equations and concepts connect \\
2D Discussed purpose of equation and how its parts interact \\
2E Recommended resources to decode content \\
2F Provided access to information in non-dominant language \\
2G Allowed students to discuss in non-dominant language \\
2H Instructor used multiple means to represent content \\
\hline \multicolumn{1}{c}{ Guideline 3: Options for comprehension } \\
\hline 3A Clicker questions cover previous content \\
3B Think/pair/share \\
3C Asked for predictions \\
3D Provided outline of big concepts \\
3E Provided agenda for class period \\
3F Formatting highlighted key information \\
3G Questions that apply new concepts \\
3H Check-ins for students to assess their understanding \\
3I Provided graphic organizers \\
3J Provided list of problem-solving steps \\
3K Modeled metacognitive behaviors for problem solving \\
3L Provided analogies \\
3M Showed real-world situations where content occurs \\
3N Scaffolded learning new content \\
\hline \hline
\end{tabular}

(e.g., Physics 3). We recruited participants from our university and professional networks, which may result in shared unconscious assumptions remaining unexamined. We did not attend to aspects of the participants' identities beyond their discipline, which leaves ways that the suggested practices operate differently along dimensions of power unexamined. We did not recruit disabled learners, an essential stakeholder group; the impact of the practices on disabled learners must be explored in future work.

Interviews were conducted remotely via Zoom by E.S. Interviews were audio-recorded and transcribed using automatic transcription via Rev.com and edited by J.J.C. All participants were asked about their initial thoughts about the protocol and for examples of accessible and inclusive practices in postsecondary STEM. In most interviews, the interviewer went through specific principles and instructional practices (although there was rarely time to discuss all items with a single participant) with prompts that varied slightly across the populations. STEM instructors were asked whether they used the exemplar practices and what those practices looked like in their instruction, DBER participants were asked whether the practices were observable and applicable to their discipline, and disability experts were asked whether the practices aligned with UDL. Additionally, each interview had a few population-specific prompts. For example, disability experts were asked how their definitions of accessibility and inclusion compared to those our team developed to guide the protocol development.

We used two analysis streams. During the interviews, E.S. took extensive notes about the participants' responses. J.J.C. conducted content analysis $[25,26]$ on the transcripts. We then compared findings about convergent (i.e., ideas that were shared by multiple participants) and divergent (i.e., ideas that appeared unique to individual participants) themes. J.J.C and E.S. discussed the implications of both convergent and divergent themes for the UDL-IPOP's possible utility across the three populations. Finally, J.J.C. returned to the content analysis notes and written transcripts to describe the themes and implications.

\section{INTERVIEW THEMES}

The majority of the non-DBER postsecondary STEM instructors $(5 / 6)$ expressed interest in the UDL-IPOP as a tool for their instructional design. For example, Physics 2 stated, "Having something like this available before you teach a class was super interesting because you can sort of say, 'Am I doing these things?'”. We present the UDLaligned instructional practices and how the interviewees described them to highlight how STEM instructors could implement UDL-aligned instructional practices.

\section{A. Varied levels of familiarity with instructional practices across guidelines and communities}

UDL Principle 1 describes multiple means of representing course content to support variation in how learners perceive and comprehend presented information. The instructional practices aligned with Principle 1 in the UDL-IPOP are displayed in Table I. Guideline 1 describes options for perception, e.g. flexible content that does not depend on a single sense like sight or hearing. While reviewing the UDL-IPOP, STEM instructors discussed providing course materials online in the learning management system (LMS; 1A), providing verbal descriptions of images (1E), and using demonstrations to support course concepts (1C). Few STEM instructors had experience allowing for customization of visual information (1B), providing captions for information presented auditorily (1D), or providing alternative text for information presented visually (1F); a possible reason is discussed in Section V.B. Guideline 2 describes options for language, mathematical expressions, and symbols to support learners in creating a shared understanding. STEM instructors described naming and defining symbols (2A) and describing connections between equations and concepts (2C). However, few had experience supporting students to build understanding in a non-dominant language $(2 \mathrm{~F}, 2 \mathrm{G})$, such as Spanish or American Sign Language. To do this, instructors could define vocabulary in multiple languages (2B), recommend 
resources for students to interpret course content (2E), or use multiple representations (e.g., words, diagrams, videos) $(2 \mathrm{H})$. Guideline 3 describes options for comprehension to support learners in generating new understanding. STEM instructors discussed providing online quizzes and selfdirected resources for prerequisite course material $(3 \mathrm{G}, 3 \mathrm{H})$, asking students to make predictions about the outcome of real-world phenomena (3C), listing learning objectives in the syllabus (3D), and connecting course content to the real world (3M). However, few instructors described providing a daily agenda that students could refer to during class or providing materials before class to support strategies students may use for full access, such as learning new vocabulary (3E) or other strategies to help students scaffold the new content they are learning $(3 \mathrm{~F}, 3 \mathrm{I}, 3 \mathrm{~J}, 3 \mathrm{~K}, 3 \mathrm{~L}, 3 \mathrm{~N})$.

UDL Principle 2 describes multiple means of allowing learners "to navigate the learning system and express what they know" [1]. The instructional practices aligned with Principle 2 in the UDL-IPOP are displayed in Table II. STEM instructors asked for clarification of the terminology in this section and did not have ideas about what many of the checkpoints would look like in practice. Guideline 4 describes options for how learners interact with course materials and tools. Instructors discussed providing students a variety of assignments (4B), allowing students to submit assignments through the LMS or email (4A), and whether to assign students to groups or allow students to selfselect groups (4C). No STEM instructors reported experience using assistive technology with students (4D), while the disability experts discussed that instructors should model how to interact with the assistive technology built into many tools we already use (i.e., Microsoft Office, Google, data acquisition tools). STEM instructors discussed few practices aligned with Guidelines 5 or 6 . Guideline 5 describes options for how learners communicate and express their developing understanding. Instructors could allow students to present (5A) and be assessed on (5D) their understandings in multiple ways, provide options for lab equipment (5B) and analytic tools (5C), and model multiple, differing ways to solve problems (5E). Guideline 6 describes options for how learners develop and implement plans for learning and monitor their learning progress. Our research collaboration had not explicated any practices aligned with Checkpoint 6.3 "Facilitate managing information and resources"; however, the UDL experts described several, including using the LMS to provide information before class and to lay out the structure of the class, such as deadlines and suggested timelines, and using a personalized folder system to provide materials directly to individual students. Instructors could also directly ask students to explain their reasoning (6D), model productive ways of thinking about problem-solving and course content $(6 \mathrm{E})$, and/or prompt students to assess their progress over time $(6 \mathrm{G})$.

UDL Principle 3 describes multiple means of engaging and motivating learners. The instructional practices aligned with Principle 3 in the UDL-IPOP are displayed in Table III.
TABLE II. Strategies to Provide Multiple Means of Action and Expression

\begin{tabular}{l}
\hline \multicolumn{1}{c}{ Guideline 4: Options for physical action } \\
\hline 4A Provided options for submission mechanism \\
4B Provided options for hands-on activities \\
4C Allowed variety of roles/tasks within groups \\
4D Discussed assistive technologies \\
\hline Guideline 5: Options for expression and communication \\
\hline 5A Provided options of how to present understandings \\
5B Variety of lab equipment available \\
5C Provided options for analytic tools \\
5D Provided variety of means of evaluation \\
5E Modeled different approaches to solve problems \\
\hline \multicolumn{1}{c}{ Guideline 6: Options for executive functions } \\
\hline 6A Provided examples of the process and product of \\
goalsetting \\
6B Posted objectives in an obvious place \\
6C Supported students' choice in engaging with course \\
6D Embedded prompts to explain your reasoning \\
6E Modeled productive thought processes \\
6F Students answer questions covering new content \\
6G Provided checkpoints to assess progress \\
\hline \hline
\end{tabular}

Guideline 7 describes options for increasing learners' interest in course content. Instructors discussed challenges providing students with options and autonomy (7A, 7B, 7C) in large enrollment courses. While some STEM instructors expressed that their discipline made relevant connections to students' lives and interests (e.g., physics textbooks that described concepts in the context of medical devices; 7D), one disability expert cautioned that instructors should critically reflect on their positionality when assessing the relevancy of examples for all students. There were a variety of opinions about the instructional practices suggest for Checkpoint 7.3 "Minimize threats and distractions", which require deeper discussion than possible in this paper $(7 \mathrm{~F}$ through 7J). Guideline 8, which describes options for supporting learners to sustain effort and overcome challenges, was not discussed with many instructors. Instructors could provide questions aligned with specific learning outcomes (8A) in a range of complexities (8C), provide opportunities for $(8 \mathrm{D}, 8 \mathrm{E})$ and scaffolding of group work $(8 \mathrm{~F}, 8 \mathrm{G})$, and focus feedback on improvement rather than on reaching a set standard $(8 \mathrm{~J})$. Guideline 9 describes options for emotionally supporting students. Instructors discussed emphasizing that students' value was not connected to their course performance (9D), but that this practice typically happened one-on-one in office hours rather than during class. Instructors could also promote a culture of healthy processing of negative emotions such as frustration or boredom (9B) and emphasize that success is about progression over time rather than intelligence (9A).

\section{B. Conflicting definitions of accessible}

Working across postsecondary STEM and UDL, we frequently encounter disciplinary differences in definitions for common terminology (e.g., see Sec IIB of Ref. 20). In 
TABLE III. Strategies to Provide Multiple Means of Engagement

Guideline 7: Options for recruiting interest

7A Provided flexibility in how to complete task

7B Provided tasks that have few restrictions

7C Provide a choice of which problems to solve

7D Discussed how concept relates to everyday life

7E Provided multiple solutions to solve problems

$7 \mathrm{~F}$ Restricted the use of cellphones in class

$7 \mathrm{G}$ Set norms of pronoun usage in class

$7 \mathrm{H}$ Made encouraging statements to class

7I Normalized error

7J Provided breaks during class

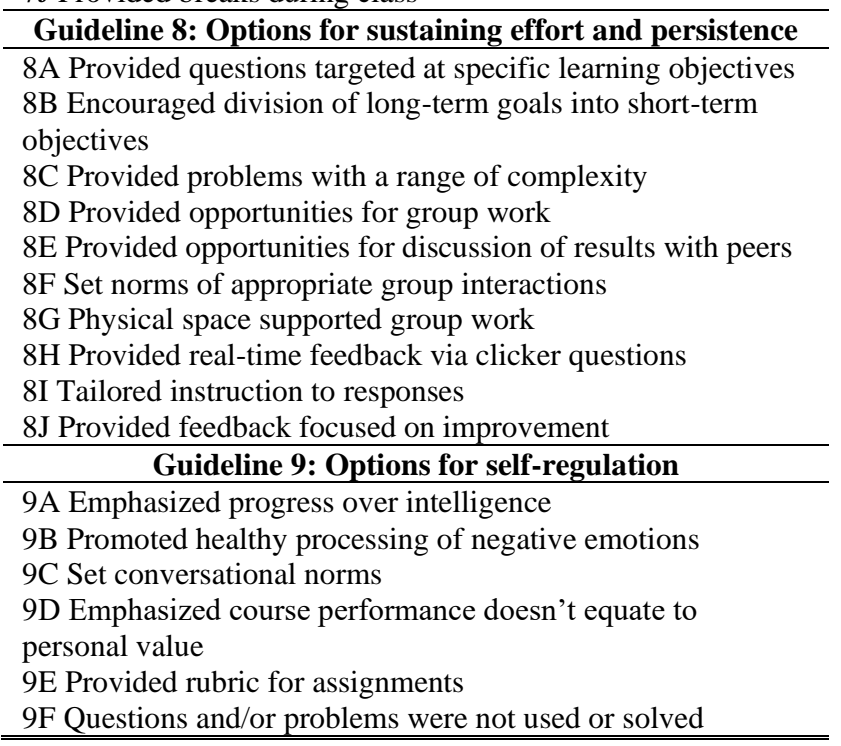

this study, we identified multiple ways participants operationalized "accessible" and "inclusive". These terms have a specialized meaning in the context of UDL. Across disciplines, participants discussed "availability" as an aspect of accessibility. For example, Disability 1 began, "accessible to me is making sure the content and materials... that all students have access to it." For some STEM instructors, accessibility ended with availability. For example, Physics 3 explained, "Accessible is... I post [in the LMS] all the material that they need... to succeed in the course... those are the things that I would say give them access because they don't have to really be in the course." We interpret this as a lay definition of accessible as "able to access" without specific attention to variation in learners' needs, abilities, and interests. Not surprisingly, each of the disability experts spoke explicitly about the need to provide access across such variations. For example, Disability 1 's response continued to describe that accessibility applied to both students with and without disabilities, with specific examples of blind students and deaf students. Similarly, Disability 3 described "accessible would mean that is available to people with varied abilities... including a diverse range of students with varied physical, cognitive and social abilities."

While this difference is not surprising, it is likely related to who these two populations have in mind when they consider if students are "able to access" the instructional environment and may explain why STEM instructors had little experience with some of the practices (e.g., 1B, 1D, 1F, 4d). For example, UDL Checkpoint 1.3 focuses on alternatives for visual information (1D, 1E). When asked about this checkpoint, STEM instructors discussed practices such as providing information outside of class, accompanying pictures with descriptive text, verbally describing visual information, recommending multiple textbooks, and introducing concepts through both lecture slides and demonstrations. Overall, it seems like the STEM instructors were considering students who are "auditory learners" as opposed to "visual learners" or students who prefer to see multiple examples of the same concept. Additionally, most of the STEM instructors did not seem to be familiar with the terminology "alternative text" as the written description of an image that would be read by a screen reader. On the other hand, some of the disability experts centered students with low incidence access needs. For example, Disability 2 said "You don't have anything for alternatives for visual. You just have text and material... what if I'm blind and deaf, you don't have anything for that...". This participant frequently discussed the need for tactile instructional approaches for students who are deafblind as well as ways to interact with students who use augmentative and alternative communication devices, such as an iPad program or "sip-and-puff" device. This finding indicates STEM instructors may need to broaden their ideas about the dimensions along which learners vary.

\section{SUMMARY AMD IMPLICATIONS}

People vary in their needs, abilities, and interests. While the UDL framework provides ideas to design instructional environments that support this learner variation, STEM instructors need professional development tools that they can understand. STEM instructors also need to gain awareness of and fluency with addressing ableism endemic in higher education [27]. We designed the UDL-IPOP to explicate instructional practices we have observed, or expect can be implemented, in postsecondary STEM courses.

In this project we found that working with a collaboration of experts from multiple disciplines supported more useful identification and description of possible UDL-aligned instructional practices. We encourage both STEM instructors and discipline-based education researchers to seek collaborations with experts outside of their home discipline(s) (e.g., exception education experts) to challenge the lenses through which we typically perceive learner variation. We intend to continue working with scholars from multiple disciplines and plan to pilot the UDL-IPOP in STEM courses in the future.

\section{ACKNOWLEDGMENTS}

This work is supported in part by National Science Foundation DUE 1612009. 
[1] CAST, Universal Design for Learning Guidelines version 2.0. Wakefield, MA: Author (2011).

[2] Thompson, A. R., Bethea, L., \& Turner, J. (1997). Faculty Knowledge of Disability Laws in Higher Education: A Survey. Rehabilitation counseling bulletin, 40(3), 166-80.

[3] Zhang, D., Landmark, L., Reber, A., Hsu, H., Kwok, O. M., \& Benz, M. (2010). University Faculty Knowledge, Beliefs, and Practices in Providing Reasonable Accommodations to Students with Disabilities. Remedial Spec. Educ. 31(4): 276286. (edited)

[3] Baker, K. Q., Boland, K., \& Nowik, C. M. (2012). A Campus Survey of Faculty and Student Perceptions of Persons with Disabilities. Journal of Postsecondary Education and Disability, 25(4), 309-329.

[5] Evans, N. J., Broido, E. M., Brown, K. R., \& Wilke, A. K. (2017). Disability in Higher Education: A Social Justice Approach: John Wiley \& Sons.

[6] Leyser, Y., Vogel, S., Wyland, S., \& Brulle, A. (1998). Faculty attitudes and practices regarding students with disabilities: Two decades after implementation of Section 504. Journal of Postsecondary Education and Disability 13(3): 5-19.

[7] Reed, M., Lund-Lucas, E., \& O'Rourke, K. (2003). Standards of Practice in Postsecondary Special Needs Programming: Student and Administrator Opinion. Canadian Journal of Higher Education, 33(2), 27-56.

[8] Norman, K., Caseau, D., \& Stefanich, G. P. (1998). Teaching students with disabilities in inclusive science classrooms: Survey results. Science Education, 82(2), 127-146.

[9] S. Rao, Faculty attitudes and students with disabilities in higher education: A literature review, Coll. Stud. J 38, 191 (2004).

[10] E. Schoen, M. Usyal, and C. D. McDonald, Attitudes of faculty members toward treatment of disabled students reexamined, Coll. Stud. J. 21, 190 (1986).

[11] M. L. Lewis, Faculty attitudes toward persons with disabilities and faculty Attitudes toward to accommodate students with learning disabilities in the classroom, Dissertation, Auburn University (1998).

[12] M. E. Skinner, Faculty willingness to provide accommodations and course alternatives to postsecondary students with learning disabilities. International Journal of Special Education 22, 32 (2007).

[13] E. M. Scanlon and J. J. Chini, Ability profiles: A framework for conceptualizing dimensions of ability, 2018 PERC Proceedings [Washington, DC, August 1-2, 2018], edited by A. Traxler, Y. Cao, and S. Wolf, DOI: 10.1119/perc.2018.pr.Scanlon.

[14] J. J. Chini and E. Scanlon, Designing for difference: Conceptualizing and planning for variations in learners' needs, abilities, and interests, AAAS IUSE blog (2021).

[15] E. M. Scanlon and J. J. Chini, Physics instructors' views about supporting learner variation: Modifying the Inclusive Teaching Strategies Inventory, 2019 PERC Proceedings [Provo, UT, July 24-25, 2019], edited by Y. Cao, S. Wolf, and M. B. Bennett, doi: 10.1119/perc.2019.pr.Scanlon.

[16] J. Schreffler, E. Vasquez III, W. James, and J. Chini, Using Observations of Universal Design for Learning to Enhance Post-secondary STEM Teaching Practices, presented at the Physics Education Research Conference 2017, Cincinnati, OH, 2017, doi: https://doi.org/10.1119/perc.2017.pr.085
[17] W. James, J. H. Cooney, J. J. Chini, E. Vasquez III, and J. Schreffler, Using Universal Design for Learning to Support Students with Disabilities in a SCALE-UP Course, The Physics $\begin{array}{lllll}\text { Teacher } & 59, & 320 & \text { (2021). }\end{array}$ https://doi.org/10.1119/10.0004878

[18] J. D. Basham, J. E. Gardner, S. J. Smith, Measuring the Implementation of UDL in Classrooms and Schools: Initial Field Test Results, Remedial and Special Education, 41, 4 (2020). doi: https://doi.org/10.1177/0741932520908015

[19] D. Fogarty, Universal Design for Learning: A New Clinical Practice Assessment Tool Toward Creating Access and Equity for ALL Students, (2017). LMU/LLS Theses and Dissertations. 475. https://digitalcommons.lmu.edu/etd/475

[20] W. James, C. Bustamante, K. Lamons, E. Scanlon, and J. J. Chini, Disabling barriers experienced by students with disabilities in postsecondary introductory physics, PRPER $\mathbf{1 6}$ 02011 (2020). doi: 10.1103/PhysRevPhysEducRes.16.020111

[21] W. James, K. Lamons, R. Spilka, C. Bustamante, E. M. Scanlon, and J. J. Chini, Hidden walls: STEM course barriers identified by students with disabilities, 2019 PERC Proceedings [Provo, UT, July 24-25, 2019], edited by Y. Cao, S. Wolf, and M. B. Bennett, doi: 10.1119/perc.2019.pr.James

[22] E. Scanlon, J. Schreffler, W. James, E. Vasquez, and J. J. Chini, Postsecondary physics curricula and Universal Design for Learning: Planning for diverse learners, Phys. Rev. Phys. Educ. Res. 14, 020101 (2018). doi: 10.1103/PhysRevPhysEducRes.14.020101.

[23] E. Scanlon, T. Legron-Rodriguez, T. Schreffler, E. Ibadlit, E. Vasquez, and J. J. Chini, Postsecondary chemistry curricula and universal design for learning: Planning for variations in learners' abilities, needs, and interests, Chem. Educ. Res. Pract. 19, 1216 (2018). DOI: 10.1039/C8RP00095F

[24] Based on prior experience, we expect that some UDL-aligned practices will not be captured by observing the classroom session. Thus, we created before and after class questions for the instructor that will be included in the full UDL-IPOP. However, discussion of the before and after class items is beyond the scope of this paper.

[25] Downe-Wamboldt, B. (1992). Content analysis: Method, applications, and issues. Health Care for Women International, 13(3), 313-321. doi: 10.1080/07399339209516006

[26] Neuendorf, K. (2002). The Content Analysis Guidebook. Thousand Oaks, CA, Sage Publications.

[27] J. T. Dolmage, Disability and higher education. University of Michigan Press. (2017) doi: 10.3998/mpub.9708722. 